Ann. Zootech., I97I, 20 (3), 305-320.

\title{
UTILISATION DES FOURRAGES GRANULÉS PAR LA CHÈVRE LAITIÈRE
}

\author{
P.-M. FEHR \\ Laboratoive de Recherches de la Chaire de Zootechnie, I. N. R. A., \\ Institut national agronomique, \\ 16, Rue Claude-Bernard, 75 - Paris (5e)
}

\begin{abstract}
RÉSUMÉ
Dans trois expériences on a étudié les effets du foin de luzerne condensé et de la luzerne déshydratée condensée et compactée distribuée en remplacement des tourteaux de l'aliment concentré ou du foin long de la ration, sur le comportement, la consommation alimentaire, la production et la composition du lait de chèvres. Tous les régimes contiennent de la paille non broyée et sont isoénergétiques.

La présence de luzerne agglomérée dans le régime diminue de $25 \mathrm{p}$. Ioo le temps consacré à la prise des repas et à la rumination, alors que le temps de repos augmente dans les mêmes proportions. Quelques chèvres éprouvent des difficultés à s'habituer au fourrage aggloméré. La présentation de la luzerne sous forme agglomérée tend à augmenter la consommation de paille et à diminuer celle d'aliments concentrés.

La production laitière est peu influencée par la forme physique de la luzerne et semble plus sensible au niveau de consommation de ce fourrage. Le taux butyreux a tendance à diminuer d'environ $2 \mathrm{~g}$ par $\mathrm{kg}$ de lait, la teneur en matières azotées à augmenter de $\mathrm{I}, 5 \mathrm{~g}$ en présence de luzerne déshydratée condensée ou compactée.

Ainsi, la luzerne agglomérée en présence de paille peut remplacer le foin long ou les tourteaux de l'aliment concentré dans la ration sans modifier sensiblement les performances laitières des chèvres.
\end{abstract}

\section{INTRODUCTION}

D'après de nombreux travaux qui ont fait l'objet d'articles de synthèse de la part de MoORe (I964), Journet et Hoden (I968) et Colin (I968), la luzerne présentée sous forme granulée offre un double intérêt ; son niveau d'ingestion est en général plus élevé que celui d'un foin normal et sa valeur nutritive notamment en énergie et en matières azotées est supérieure. Toutefois, elle peut avoir aussi des inconvénients comme, entre autres, abaisser le taux butyreux ou dans certains cas perturber l'état de santé. Ainsi, il nous est apparu utile, en s'inspirant des études dont les résultats ont été précédemment bien établis sur vaches, de préciser la valeur alimentaire de la luzerne granulée pour la chèvre laitière et la manière la plus satisfaisante de l'introduire dans le régime.

La chèvre a une aptitude à consommer de grandes quantités de fourrages 
(FEHR et DISSET, I969). Par ailleurs, pour couvrir des besoins de production souvent élevés, son régime contient en général une forte proportion d'aliments concentrés. Dans ces conditions, au cours de trois expériences, nous avons successivement cherché à savoir si les granulés de luzerne peuvent remplacer d'abord les tourteaux de l'aliment concentré, puis le foin long de luzerne et enfin à la fois les tourteaux et le foin long dans la ration. Différents types de granulés de luzerne ont été employés : dans les deux premières expériences, du foin de luzerne sous forme condensée (broyé et aggloméré), et dans la troisième de la luzerne déshydratée sous forme condensée et compactée (agglomérée sans subir au préalable un broyage).

\section{MATÉRIEL ET MÉTHODES}

\section{I. - Dispositif expérimental et régimes (cf. tabl. I et 2)}

Expérience $I$ : Foin de luzerne condensé utilisé en remplacement des tourteaux de l'aliment concentré.

Douze chèvres de race Alpine ayant en moyenne quatre-vingt-dix jours de lactation au début de l'essai, sont réparties en deux lots équilibrés en production laitière et en taux butyreux. Elles consomment successivement les deux régimes : T et $\mathrm{L}$, comme l'indique le tableau i au cours de trois périodes de 28 jours dont les 14 premiers sont considérés comme période de transition.

TABLEAU I

Dispositifs expérimentaux

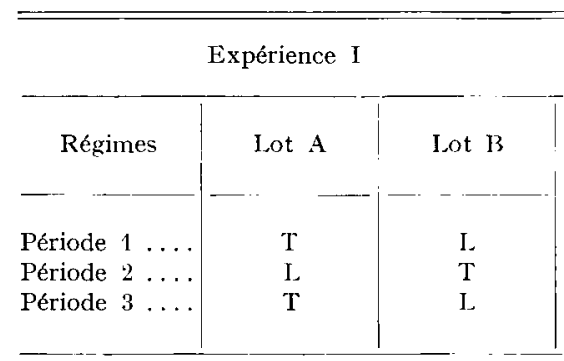

$\mathrm{T}$ : Régime témoin avec tourteaux.

L : Régime avec foin de luzerne condensé.

Expérience II

\begin{tabular}{|c|c|c}
\hline Régimes & Lot A & Lot B \\
\hline & & \\
Période 1... & $\mathrm{C}$ & $\mathrm{F}$ \\
Période 2... & $\mathrm{F}$ & $\mathrm{C}$ \\
Période 3... & $\mathrm{C}$ & $\mathrm{F}$ \\
\hline
\end{tabular}

G : Régime avec foin de luzerne condensé.

Ii : Régime avec foin de luzerne long.

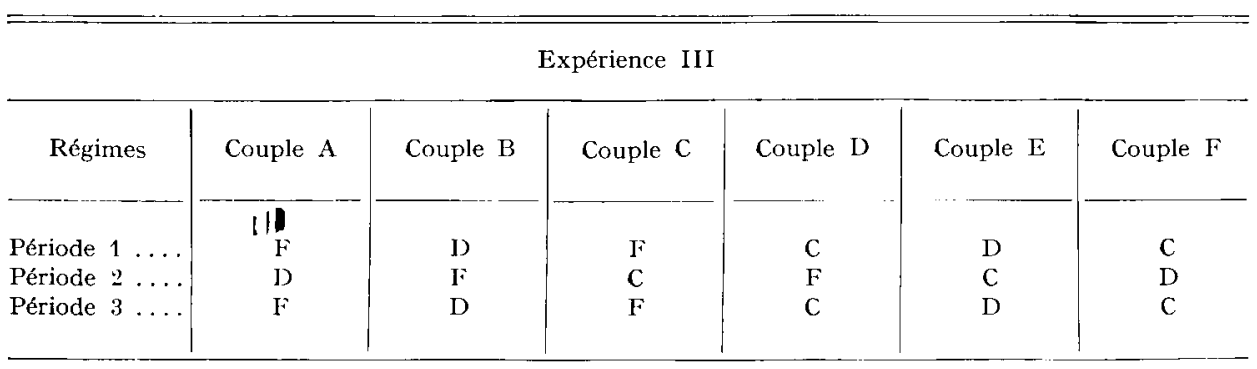

F : Régime avec foin de luzerne long.

I : Régime avec luzerne déshyđtatée condensée.

C : Régime avec luzerne déshydratée compactée. 
Le régime $T$ est composé də foin de pré, d'un aliment concentré énergétique à base d'orge, d'avoine, de pulpes de betteraves déshydratées, d'un c smposé minéral vitaminique et d'un aliment concentré azoté à base de tourteaux de lin et d'aras'ide. Dans le régime L l'aliment concentré azoté est remplacé par du foin de luzerne condensé la première coupə, récolté au stade pleine floraison, broyé avec un arille à mailles de $3 \mathrm{~mm}$ et aggloméré dans une presse à filières de $5 \mathrm{~mm}$. En outre, les chèvres pəuvent consommer un peu de paille de leur litière.

Les valeurs énergétiques et azotées des différents aliments calculées à partir de l'analyse fourragère effectuée au laboratoire, sont rapportées au tableau 2. Il faut remarquer que les valeurs obtenues pour la luzerne condensée sont probablement assez approximatives en raison de notre manque de connaissances sur sa digestibilité réelle.

TABLEAU 2

Valeur énergétique et azotée des aliments utilisés

\begin{tabular}{|c|c|c|}
\hline Aliments & U.F./kg & M.A.D. $(g / k g)$ \\
\hline 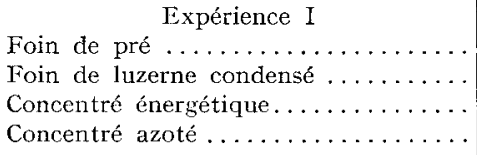 & $\begin{array}{l}0,48 \\
0,50 \\
0,86 \\
0,88\end{array}$ & $\begin{array}{r}48 \\
122 \\
58 \\
219\end{array}$ \\
\hline $\begin{array}{l}\text { Expérience II } \\
\text { Paille de blé } \ldots \ldots \ldots \ldots \ldots \ldots \\
\text { Foin de luzerne long } \ldots \ldots \ldots \ldots \ldots \\
\text { Foin de luzerne condensé } \ldots \ldots \ldots \ldots \\
\text { Concentré énergétique } \ldots \ldots \ldots \ldots \ldots \\
\text { Concentré lactation } \ldots \ldots \ldots \ldots \ldots\end{array}$ & $\begin{array}{l}0,29 \\
0,35 \\
0,39 \\
0,87 \\
0,95\end{array}$ & $\begin{array}{r}1 \\
75 \\
90 \\
60 \\
163\end{array}$ \\
\hline 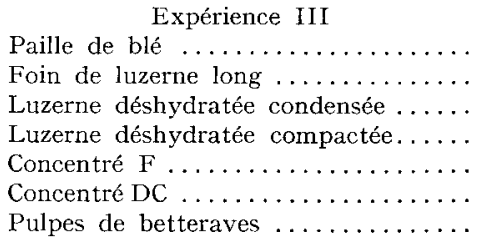 & $\begin{array}{l}0,28 \\
0,45 \\
0,58 \\
0,56 \\
0,90 \\
0,90 \\
0,82\end{array}$ & $\begin{array}{r}1 \\
104 \\
155 \\
146 \\
107 \\
82 \\
43\end{array}$ \\
\hline
\end{tabular}

La ration de chaque chèvre est établie suivant les recommandations de FEHR et Disset (1969). Elle comprend toujours un $\mathrm{kg}$ de foin et une quantité d'aliment concentré établie suivant le niveau de production au début de la première période et maintenue constarite pendant toutel'expérience. Quel que soit le régime, une chèvre, pour le même bosoin, reçoit la même quantité d'unités fourragères (U.F.) et de matières azotées digestibles (M.A.D.). La luzerne condensée et l'aliment azoté renferment les mêmes quantités de M.A.D. par U.F. Ainsi le remplacement de l'aliment concentré azoté par la luzerne condensée peut s'effectuer dans un rapport pondéral de 4 à 7 de façon isoénergétique et isoazotée.

Deux repas de foin et deux repas d'aliments concentrés sont distribués quotidiennement. L'aliment concentré azoté ou la luzerne condensée sont présentés en mélange avec l'aliment concentré énergétique. Les changements de régime s'étalent sur quatre jours.

Expérience II : Foin de luzerne condensée utilisé en remplacement du foin de luzerne normal.

L'expérience II est réalisée suivant un dispositif expérimental et des conditions semblables à celles de l'expérience $I$, à quelques exceptions près. Les douze chèvres sont placées sur caillebotis et ne peuvent pas consommer de paille autre que celle distribuée dans la mangeoire.

Les animaux reçoivent $1,2 \mathrm{~kg}$ de foin de luzerne de première coupe récoltée en fin de floraison présenté sous forme longue (régime F) ou sous forme condensée (régime C). Dans ce dernier cas, le foin est broyé à la grille de $3 \mathrm{~mm}$ et mis en bouchons de $5 \mathrm{~mm}$. Ces foins de luzerne présentés 
différemment proviennent du même champ et ils sont récoltés dans les niêmes conditions. Leur qualité médiocre est due aux mauvaises conditions atmosphériques de l'année (tabl. 2). Les deux rations de base sont complétées avec $0,5 \mathrm{~kg}$ de paille de blé de bonne qualité. Un aliment concentré énergétique à base de céréales, de pulpes de betteraves, et d'un composé minéral vitaminique, rééquilibre la ration excédentaire en matières azotées pour une production laitière de I,5 kg de lait. Au-dessus de cette production, un aliment concentré "lactation s à base d'orge et de tourteau de soja est consommé par les chèvres. La quantité distribuée de cet aliment est calculée suivant le niveau de production et réajustée au début de chaque période.

Expérience III : Luzerne déshydratée condenséc et compactéo utilisée en remplacement du foin normal et des tourtcaux de l'aliment concentré.

Six couples de deux chèvres comprenant une adulte et une primipare sont constitués de façon à être aussi comparables que possible en production laitièrc et en conıposition du lait. Les chèvres consomment les trois régimes expérimentaux $\mathrm{F}, \mathrm{D}$ et $\mathrm{C}$ pendant trois périodes de 28 jours suivant le dispositif expérimental indiqué au tableau $\mathbf{I}$.

Le régime $F$ comprend du foin de luzerne entier ( $2^{e}$ coupe, stade pleine floraison) de bonne qualité (tabl. 2) et un aliment concentré $\mathrm{F}$ à base de céréales, de tourteau de soja et d'un composé minéral vitaminique. Le régime $\mathrm{D}$ renferme de la luzerne déshydratée condensée ( $2^{\mathrm{e}}$ coupe, début floraison) en bouchons de $5 \mathrm{~mm}$ qui a subi un broyage à la grille de $2 \mathrm{~mm}$, un aliment concentré $\mathrm{DC}$ à base de céréales exclusivement et d'un composé minéral vitaminique. Dans le régime $\mathrm{C}$ la luzerne déshyđratée ( $2^{\mathrm{e}}$ coupe, début floraison) est présentée sous forme compactée,agglomérée par une presse à filières de Io $\mathrm{mm}$, sans broyage préalable. En réalité, un broyage partiel a lieu lors du compactage et sculement $35 \mathrm{p}$. I oo du poids total du produit utilisé est constitué par des particules d'une longueur supérieure à $0,5 \mathrm{~mm}$. En outre, tous les régimes sont complétés par $0,3 \mathrm{~kg}$ de paille de blé et par 0,5 $\mathrm{kg}$ de pulpes de betteraves déshydratées.

Les luzernes condensées et compactées renferment plus de matières azotécs que le foin de luzerne. De ce fait, l'aliment concentré F distribué avec le foin long en contient plus que l'aliment DC, comme le montre le tableau 2 afin que pour un besoin identique les chèvres reçoivent la. même quantité de matières azotées. Par ailleurs, les produits déshydratés étant plus énergétiques que le foin, la quantité d'aliment concentré $F^{\prime}$ distribuée est toujours supérieure de o, I $\mathrm{kg}$ à celle de l'aliment DC pour un même besoin. Enfin, les rations sont réajustées en énergie en fonction des besoins au début de chaque période. Mais ellcs sont excédentaires en matières azotées surtout pour des besoins de production faibles. Nous avons conservé ce déséquilibre a fin de faire ingérer des quantités appréciables de luzerne. Il est distribué $0,8 \mathrm{~kg}$ de luzerne, quelle que soit sa forme physique, jusqu'à $3 \mathrm{~kg}$ de lait et $\mathrm{I}, 05 \mathrm{~kg}$ au-dessus de $3 \mathrm{~kg}$ de lait.

\section{2. - Mesures}

Dans toutes les expériences, les rations individuelles et les refus éventuels de foin, de paille, de pulpes et d'aliments concentrés sont pesés à tous les repas. La production laitière est enregistrée quotidiennement, le taux butyreux (méthode (ierber) trois fois par semaine et la teneur en matières azotées (méthode au noir amido) exclusivement dans les expériences II et III, une fois par semaine. Les animaux sont pesés au début et à la fin de chaque période.

En outre, dans l'expérience III, le comportement des chèvres est observé visuellement pendant une journée de chaque période, entre $6 \mathrm{~h}$ I 5 et i $8 \mathrm{~h} 45$ en clistinguant le temps passé à la consommation des repas, à la rumination et au repos.

\section{3. - Expression des résultats}

Les quantités moyennes de matières sèches d'aliments consommés quotidiennement rapportées au poids métabolique $\left(\mathrm{P}^{3 / 4}\right)$ n'apportent apparemment guère plus de renscignements que les moyennes par chèvre. Nous avons de ce fait présenté exclusivement celles-ci dans les tableaux de résultats 3,4 et 5 en mentionnant toutefois les différences de signification entre les deux modes d'expression, quand elles existent. Nous avons tenu à rapporter les quantités ingérées d'U.F. et de M.A.D. totales malgré l'imprécision de nos estimations sur la valeur énergétique et azotée des fourrages agglomérés. Étant donné que les 14 premiers jours de chaque période sont considérés comme transition, nous n'avons tenu compte dans les résultats que des données des deux dernières semaines. Enfin, la variation journalière moyenne de poids vif des chèvres cxprimée en g est la différence de poids entre le début et la fin de la période divisée par 28 jours.

Le traitement des résultats des expériences I et II est réalisé par analyse de variance. Les données de chaque lot sont interprétées séparément, suivant la méthode des blocs, en tenant compte des variations individuelles, ce qui permet d'affiner l'analyse statistique. Ce procédé évite 
de supposer que les deux lots de chèvres restent strictement comparables à tous moments de l'expérience. Les résultats globaux en réunissant les données des lots A et B ne sont rapportés dans les tableaux qu'à titre indicatif.

L'interprétation est effectuée de manière à estimer les effets des régimes, confondus a priori avec ceux du temps. Les deux degrés de liberté du facteur période sont décomposés et consacrés respectivement aux comparaisons des résultats des deux périodes extrêmes, de même régime, et des résultats de la moyenne de ces deux extrêmes à ceux de la période médiane penclant laquelle un régime différent est distribué. Cette dernière comparaison permet de tester la linéarité de l'évolution du phénomène étudié dans le temps, et la confrontation des résultats des deux lots, nourris en régimes alternés, permet ainsi d'évaluer les effets des régimes.

Une autre analyse de variance réalisée dans l'expérience III teste l'influence de trois facteurs de variation : le temps, les régimes alimentaires et les différences entre les animaux primipares et adultes. L'effet " régime " est décomposé en une comparaison entre les résultats obtenus avec les régimes $\mathrm{D}$ et $\mathrm{C}$ dont la nature est assez proche et en une autre comparaison entre les résultats du régime $F$ et ceux des régimes $D$ et $C$ pris globalement pour bien mettre en évidence les différences entre la forme longue et la forme agglomérée de la luzerne.

\section{RÉSULTATS}

Les résultats du comportement alimentaire et des performances laitières des chèvres qui ont participé aux expériences I, II et III sont respectivement rapportés dans les tableaux 3,4 et 5 .

\section{Consommation.}

\section{Expérience I}

La présence dans le régime de luzerne condensée à la place de l'aliment concentré azoté, quoique distribuée en quantité plus élevée, ne diminue pas la consommation de foin de pré. Les différences apparues significatives sur les moyennes par chèvre ne le sont plus lorsque les résultats sont rapportés au poids métabolique.

Le foin de luzerne condensé est bien accepté par la plupart des chèvres, mais trois animaux du lot $\mathrm{B}$ sont lents à s'habituer à ce type d'aliment pendant la première période, alors qu'ils le consomment de façon satisfaisante en troisième période. De plus, un animal du lot $\mathrm{A}$ a refusé pendant la seconde période près de $7^{\circ} \mathrm{p}$. Ioo de la luzerne distribuée. Cette consommation moindre de luzerne observée sur queiques animaux se répercute apparemment sur la quantité totale d'U.F. et de M.A.D. ingérées

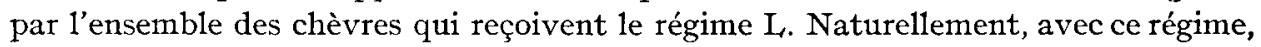
la consommation d'aliments concentrés en incluant le granulé de luzerne est supérieure à celle enregistrée avec le régime $T$, car pour apporter la même énergie, la quantité de luzerne distribuée est plus élevée que celle de l'aliment concentré azoté.

\section{Production et composition du lait.}

Dans les deux lots, les productions de lait et de matières grasses des chèvres diminuent de façon linéaire et le taux butyreux varie faiblement pendant les trois périodes successives. Aucune différence significative sur ces performances due à la nature des régimes n'a pu être mise en évidence. Ainsi, malgré une ingestion apparemment plus faible d'énergie, les chèvres consommant les granulés de luzerne ont un niveau de production très comparable à celles qui reçoivent des tourteaux. En réalité il est possible que l'effet dî aux régimes soit atténué par la légère suralimentation des chèvres en fin d'expérience. 


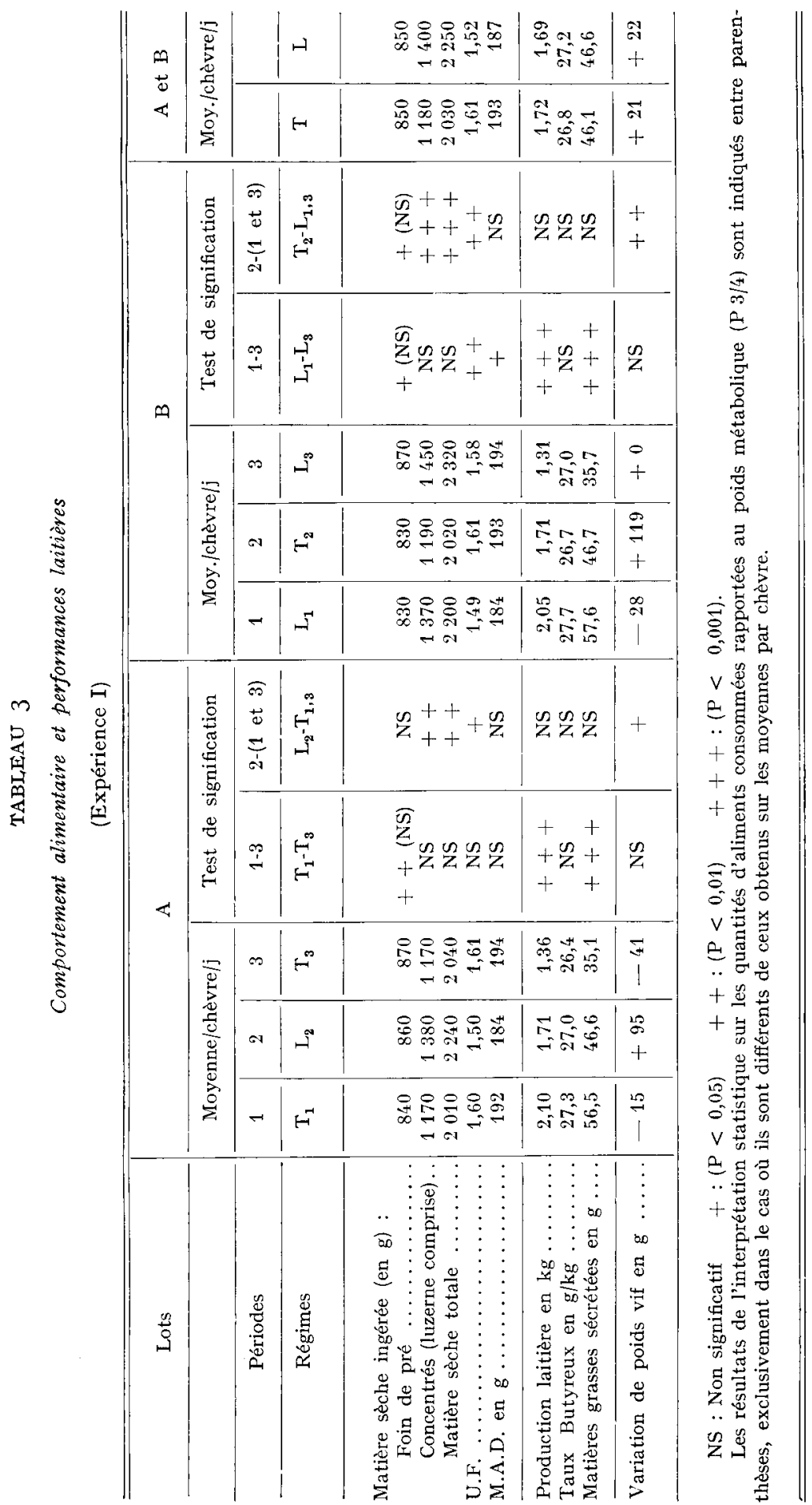




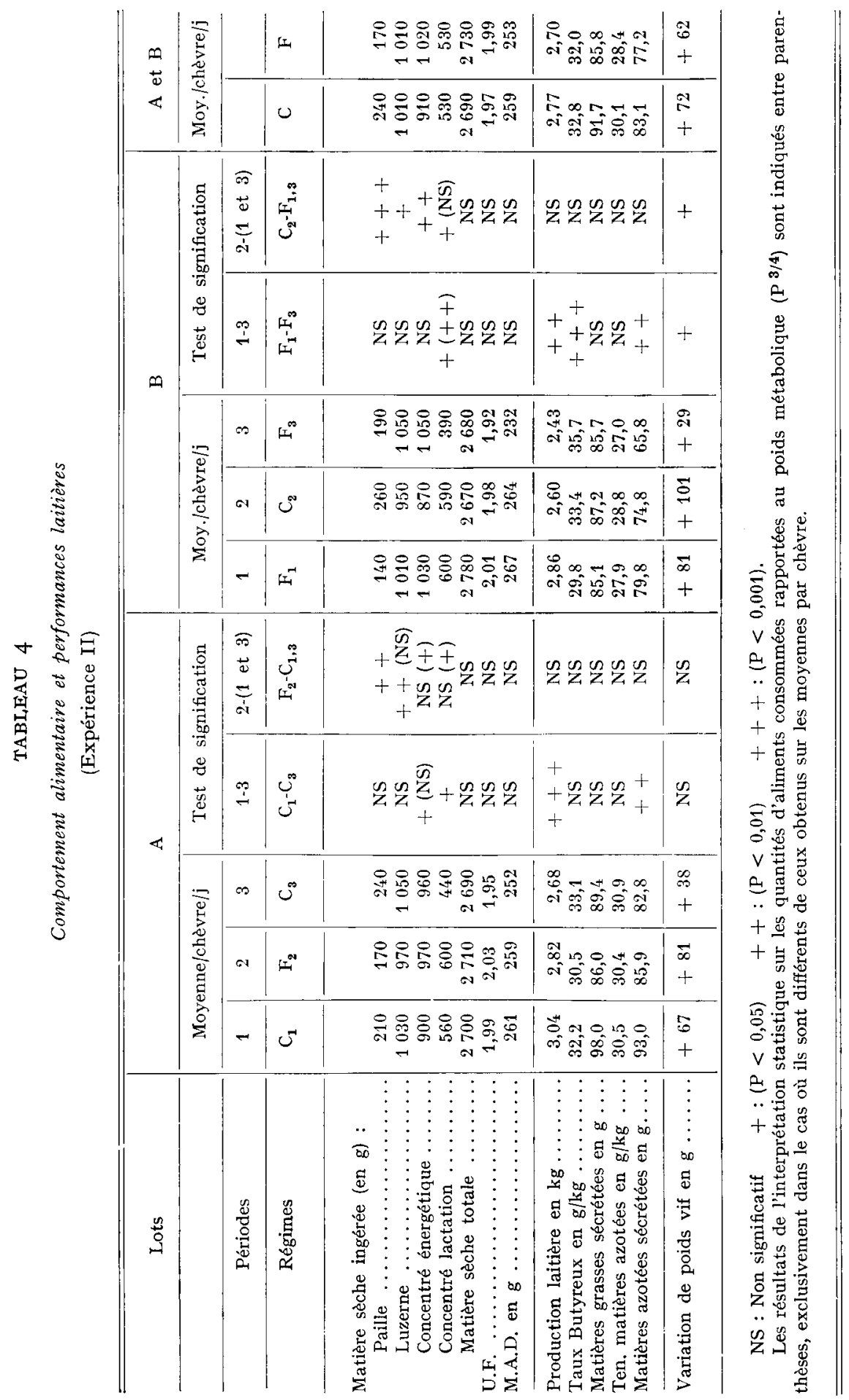




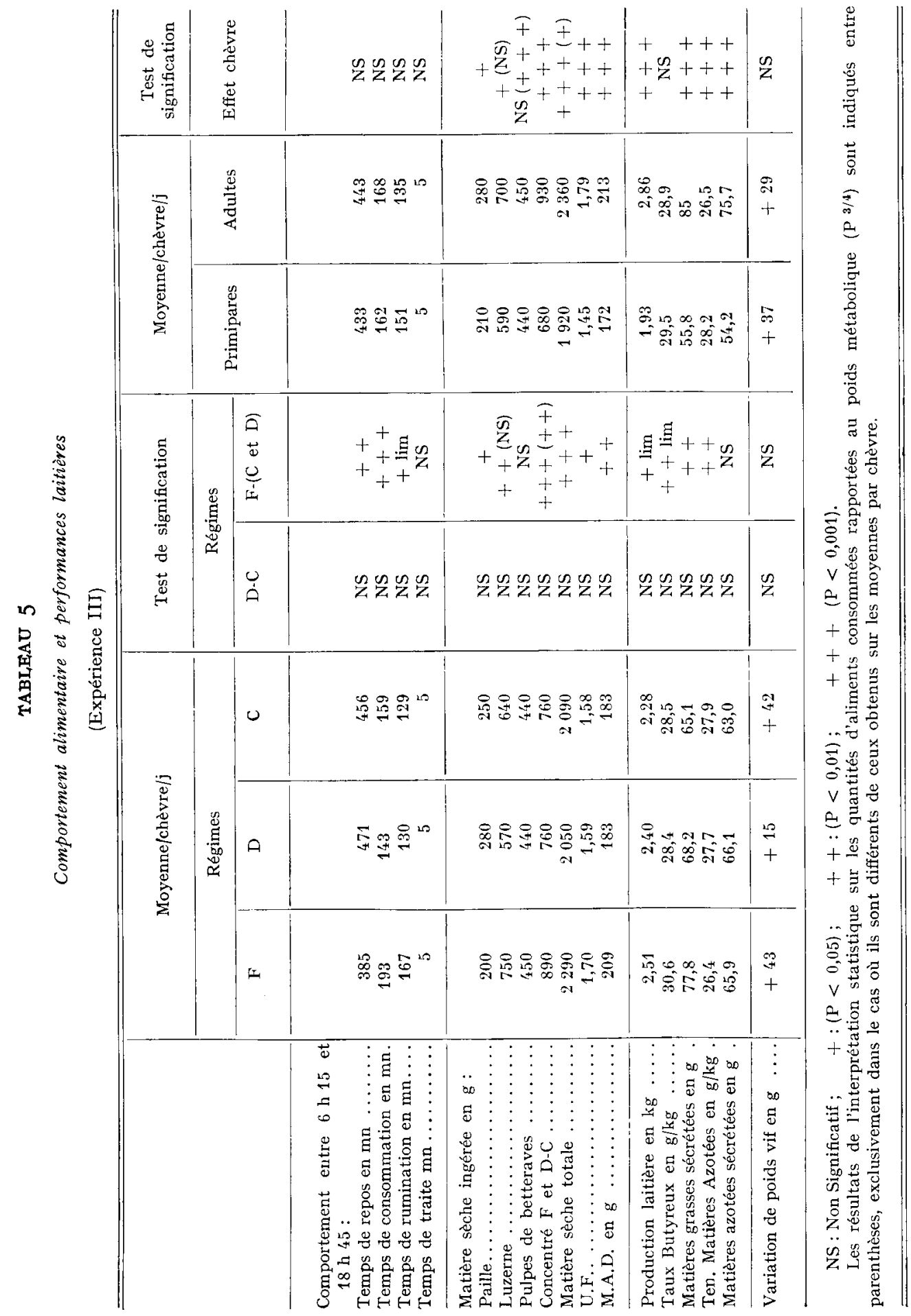


Les variations de poids vif des animaux au cours de l'expérience doivent probablement moins être attribuées à la nature du régime qu'à la période. Fin effet, quel que soit le régime, les chèvres ne gagnent du poids que pendant la seconde période sans raison bien déterminée.

\section{Expérience II}

\section{Consommation.}

Le foin de luzerne condensé est aussi bien consommé que le même foin présenté sous forme longue. Le lot $A$ a légèrement moins ingéré de foin long, alors que le résultat contraire est enregistré dans le lot $\mathrm{B}$. La présence de granulé de luzerne dans le régime a deux autres conséquences : la consommation de paille de blé non broyée augmente très nettement et celle de l'aliment concentré énergétique diminue, en particulier dans le lot $\mathrm{B}$. Cette modification du comportement alimentaire n'a que très peu d'incidence sur les quantités de matière sèche totale, d'U.F, et de M.A.D. ingérées.

\section{Production et composition du lait.}

Dans la période préexpérimentale, toutes les chèvres reçoivent un régime à base de foin de pré. Les deux lots sont alors équilibrés en production laitière et en composition du lait. L,e remplacement de ce foin de pré de bonne qualité par de la luzerne médiocre a déséquilibré les lots. La production laitière du lot $B$ recevant le foin long a baissé plus que celle du lot $\mathrm{A}$; ce qui explique que les chèvres du lot $\mathrm{A}$ ont une production sensiblement supérieure tout au long de l'expérience.

La comparaison des productions laitières, des matières grasses sécrétées, des matières azotées, du taux butyreux et de la teneur en matières azotées pendant la première et la troisième périodes d'une part, et pendant la seconde période d'autre part, ne permet pas de mettre en évidence des différences significatives. Ainsi, la nature des régimes n'a guère d'influence sur les performances laitières. Toutefois, si on considère globalement les résultats des lots $\mathrm{A}$ et $\mathrm{B}$, ces performances sont légèrement supérieures quand le foin de luzerne est présenté sous forme condensée. Par ailleurs, alors quela production laitière et la sécrétion de matières azotées des première et troisième périodes sont significativement différentes, la sécrétion des matières grasses ne l'est pas en raison, notamment, de la remontée très sensible du taux butyreux pendant la troisième période.

Le poids des chèvres s'élève constamment au cours de l'expérience. Dans le lot $B$, le gain de poids est significativement plus rapide avec la luzerne condensée et dans le lot $\mathrm{A}$, il est moins rapide avec cet aliment, mais de manière non significative.

\section{Comportement.}

\section{Expérience $I I I$}

Les temps que les chèvres consacrent à la prise des repas et à la rumination sont très différents suivant les régimes. Ils sont significativement plus élevés en présence de foin de luzerne entier dans le régime qu'avec de la luzerne granulée, qu'elle soit condensée ou compactée. En conséquence, le repos en position debout ou couchée est significativement moins long. En outre, avec la luzerne condensée, les repas sont légèrement plus rapides qu'avec la luzerne compactée mais de façon non significative. 


\section{Consommation.}

Plus de 25 p. roo de la luzerne déshydratée condensée distribuée et environ I3 p. Ioo de la luzerne compactée n'ont pas été consommés. Le foin long qui est de bonne qualité est mieux accepté bien que la différence cesse d'être significative lorsque les consommations sont rapportées au poids métabolique. Il semble que plus le granuié de luzerne renferme des particules fines, plus il risque d'être mal accepté par les chèvres et plus elles recherchent à consommer une forte quantité de paille. En effet, la consommation de paille est la plus faible quand les chèvres disposent de foin long et la plus élevée quand elles ingèrent de la luzerne condensée. Les pulpes de betteraves sont ingérées dans les mêmes proportions quel que soit le régime. La plus grande quantité d'aliments concentrés ingérée avec le régime $\mathrm{F}$ (foin de luzerne entier) s'explique en partie par la distribution supérieure de o, I $\mathrm{kg}$ pour un même niveau de production. Néanmoins, en éliminant cette cause de variation, il semble bien qu'avec ce régime, les chèvres acceptent plus facilement l'aliment concentré. En outre, l'ingestion plus faible de matière sèche totale, d'U.F. et de M.A.D. avec les régimes $\mathrm{C}$ et $\mathrm{D}$ (luzerne déshydratée compactée et condensée) est principalement due aux refus de luzerne plus importants sous forme granulée que sous forme de foin long.

\section{Production et composition du lait.}

$\mathrm{Au}$ cours de la période expérimentale, les six couples de chèvres ont des productions laitières très comparables. L'introduction de la luzerne condensée ou compactée dans le régime pendant la première période fait légèrement plus baisser le niveau de production que le foin normal. Ainsi, les couples qui reçoivent du foin long en première période, ont une production de lait moyenne au cours de toute l'expérience, un peu supérieure à celle des autres couples.

Les chèvres qui consomment du foin entier produisent plus de lait et de matières grasses. Le taux butyreux de leur lait est plus élevé, mais la teneur en matières azotées est plus faible. Par ailleurs, les performances des chèvres qui ingèrent de la luzerne condensée ou compactée ne sont pas significativement différentes. Les productions de lait, de matières grasses et de matières azotées sont légèrement moins bonnes avec la luzerne compactée qu'avec la luzerne condensée, alors que le taux butyreux et la teneur en matières azotées sont très voisins.

Les chevrettes en première lactation ont évidemment des performances laitières inférieures à celles des chèvres adultes. Leur niveau de consommation également plus faible, peut expliquer leurs performances laitières. Toutefois, le taux butyreux et la teneur en matières azotées de leur lait sont plus élevés.

Le gain de poids des chèvres est plus limité avec la luzerne condensée qu'avec les autres formes de luzerne. En ingérant du foin de luzerne long ou de la luzerne compactée, le poids des animaux augmente dans les mêmes proportions bien qu'avec la luzerne compactée, les niveaux de consommation et de production laitière soient plus faibles et atteignent même des valeurs comparables à celles enregistrées avec la luzerne condensée. La substitution de luzerne agglomérée au foin long de luzerne si elle modifiait le poids des contenus digestifs, aurait tendance plutôt à le diminuer et de ce fait à réduire le gain de poids des chèvres, comme peut-être cela se produit avec la luzerne condensée. Ainsi, la luzerne compactée pourrait avoir une légère tendance à favoriser le gain de poids des chèvres au détriment de la production laitière. 


\section{DISCUSSION ET CONCLUSIONS}

Les expériences décrites ci-dessus ont une portée limitée en raison du nombre assez faible d'animaux qui y participent. Les durées des périodes expérimentales sont trop brèves pour estimer les effets à long terme sur le gain de poids et la production de lait. Mais une expérience portant sur la production laitière peut difficilement s'étaler sur une plus longue période parce que, d'une part, les lots risqueraient d'évoluer d'une façon trop différente et que, d'autre part, la linéarité de la diminution de la production laitière dont nous avons vérifié par ailleurs l'exactitude au milieu de la lactation des chèvres, serait alors plus difficilement acceptable. Enfin, les résultats auxquels nous aboutissons ne peuvent être généralisés qu'avec prudence à des conditions expérimentales différentes des nôtres. En particulier, la luzerne en granulés n'a jamais été distribuée ad libitum comme dans la plupart des études effectuées sur ce sujet par d'autres auteurs. Au contraire, nous avons toujours rationné les animaux de façon à distribuer des rations isoénergétiques et isoazotées. En outre, nous avons utilisé des luzernes granulées avec leurs propres caractéristiques. Il n'est pas sûr que nous aurions obtenu les mêmes résultats si elles avaient présenté d'autres caractéristiques dues par exemple à un traitement technologique différent.

\section{I. - Comportement.}

Au cours de ces trois expériences, aucune chèvre, même celles qui refusent une partie de la ration, ne montre de troubles digestifs tels que des diarrhées ou des ballonnements. L'absence d'accidents de ce type est sans doute à mettre à l'actif de la paille non broyée qui est distribuée avec tous les régimes (LARvor, I967).

La substitution du foin normal par du fourrage condensé ou compacté dans le régime comme l'a montré l'expérience III, modifie très sensiblement le comportement des chèvres pendant la journée.

Bien que la réaction à ce type de régime soit variable suivant les individus, on peut estimer qu'en moyenne les temps consacrés à la prise des repas et à la rumination diminuent de 25 p. Ioo, alors que le temps de repos augmente dans les mêmes proportions. De tels résultats ont déjà été observés avec de la luzerne condensée, notamment sur moutons (KECK et coll., I962 ; RUCKEBUSCH et MaRQUET, I963; DEMARQUILLY et JOURNE'T, I967; PINOT et JARRIGE, I968) et sur vaches laitières (Journet et Demarquiliy, I967; Journet et Jarrige, i967 ; Journet, I97o). La préhension d'un fourrage sec sous forme granulée est nettement plus aisée que sous forme longue. De plus, devant un fourrage entier, les chèvres passent souvent du temps à inventorier les différents brins qui le composent avant de se décider à en consommer certains (FEHR et DISSET, I969). L'inexistence de ce comportement de la chèvre devant un produit condensé ou compacté d'aspect homogène est peut-être la cause d'une insatisfaction qui s'extériorise parfois par une certaine nervosité au moment des repas.

L'intensité de cette modification du comportement de l'animal semble liée à la proportion de particules broyées très grossièrement ou de brins longs de fourrages 
dans la ration. En effet, le phénomène est plus accusé avec la luzerne condensée qu'avec la luzerne compactée. De plus, la paille de blé non broyée paraît l'atténuer. Dans un autre essai, les chèvres qui reçoivent exclusivement de la luzerne déshydratée condensée et un aliment granulé passent un temps très court à consommer leur ration et ne présentent plus de rumination ( $\mathrm{FEHR}$, résultats non publiés).

\section{2. -... Consommation}

Ėn généra1, la luzerne sous forme granulée est assez bien acceptée par les chèvres. Néanmoins, certaines au début ont du mal à s'y habituer, d'autres persistent pendant toute l'expérience à ne l'accepter qu'en faible quantité ou font des refus périodiques. Ces constatations déjà rapportées chez la vache par Journetr et HodEn, (Ig68) qui précisent que 5 à ro p. Ioo des vaches refusent totalement ce type d'aliments, prennent une plus grande importance chez la chèvre en raison du caractère capricieux de son comportement alimentaire. L'appétibilité de la luzerne est assez médiocre surtout lorsque le broyage est relativement fin comme ce fut le cas pour la luzerne déshydratée utilisée dans l'expérience III broyée à la grille de $2 \mathrm{~mm}$. DEMARQUILLY (Ig68) note qu'en utilisant la même grille, une luzerne déshydratée est broyée plus complètement qu'un foin, car elle est en général plus sèche. Avec les autres types de luzerne, foin de luzerne condensée broyée à la grille de $3 \mathrm{~mm}$ (Expérience II) ou luzerne déshydratée compactée (Expérience III) les refus se sont maintenus dans nos conditions de distribution à un niveau de Io à I5 p. I 00 qui peut s'observer couramment avec du foin long, surtout si sa qualité est médiocre.

La dureté des aliments présentés ne semble pas avoir joué un rôle déterminant dans ces différences d'appétibilité. Ėn effet, aucun fourrage condensé distribué n'est aussi dur que les pulpes de betteraves déshydratées dont les chèvres s'accommodent fort bien. D'ailleurs, elles préfèrent généralement un aliment granulé plutôt dur que mou. Notons toutefois que la luzerne déshydratée compactée est légèrement moins dure que la luzerne condensée.

Par ailleurs, le fourrage présenté sous forme agglomérée a une incidence sur la consommation des autres aliments de la ration. Il augmente la quantité de paille non broyée ingérée, et diminue celle des aliments concentrés (Expériences II et III). D'autre part, il ne modifie pas la consommation de foin de pré lorsqu'il remplace la fraction azotée de 1'aliment concentré (Expérience I). Ainsi, d'après son comportement la chèvre semble plutôt considérer une luzerne condensée comme un aliment concentré que comme un fourrage normal, en raison probablement de son faible encombrement. Et, lorsqu'une luzerne condensée ou compactée remplace du foin long, cet animal a tendance à rééquilibrer en partie de lui-même le rapport fourrage long/aliment granulé en ingérant plus de paille et moins d'aliments concentrés; ce qui permet surtout de maintenir la rumination.

\section{3. - Production et composition du lait}

Les productions de lait ne doivent pas être comparées d'une expérience à l'autre, car les aptitudes laitières des chèvres qui participent à chacune des expériences, sont différentes.

La production laitière est assez peu influencée par la nature du régime au cours 
des trois expériences. Les variations ne sont significatives que dans 1'expérience III où le foin long de luzerne de bonne qualité donne des performances légèrement meilleures que les luzernes déshydratées condensées ou compactées ; ce qui s'explique en grande partie par une moindre consommation due aux refus plus importants de luzerne agglomérée. En revanche, lorsque le foin long est de médiocre qualité, le foin condensé consommé en quantités égales permet d'obtenir une production laitière comparable et même très légèrement supérieure (Expérience II). Ainsi les faibles variations de production laitière semblent s'expliquer plus par des différences de niveau de consommation que par la forme physique du fourrage. OwEN (Ig69) remarque aussi sur vaches laitières qu'avec une ration contenant un minimum de fourrages longs, la luzerne granulée n'a pas d'incidence sur la production laitière et qu'une diminution de celle-ci n'est due qu'à une baisse de l'énergie ingérée. JourNET et JARRIGE (I967) et JOURNET (I970) avec des rations assez différentes des nôtres, n'enregistrent pas de variations importantes de la production laitière.

Il est délicat de mettre en relation des variations observées sur la production laitière, la consommation énergétique et les différences de poids, car elles sont de faible amplitude et souvent le fait d'une ou de quelques chèvres et non de l'ensemble du lot, en particulier pour la consommation alimentaire. Néanmoins, il semble que les régimes contenant de la luzerne agglomérée ont une utilisation énergétique pour la production laitière aussi bonne sinon très légèrement meilleure que les autres régimes, sauf peut-être pour le régime à base de luzerne compactée de l'expérience III.

En présence de luzerne agglomérée dans la ration, le taux butyreux n'a baissé que dans l'expérience III, d'ailleurs dans des proportions limitées $(2 \mathrm{~g} / \mathrm{kg}$ de lait) alors que de nombreux auteurs ont observé une chute du taux butyreux souvent beaucoup plus accusée (MOORE, I964; CoLIN, I968). Cette diminution du taux butyreux inexistante dans l'expérience II et faible dans l'expérience III, est probablement due aux raisons suivantes. Les animaux ont toujours de la paille à leur disposition. Le broyage des luzernes distribuées n'est peut-être pas suffisamment fin pour provoquer des chutes importantes de taux butyreux (RODRIGUE et AL,EN, I960; PALmouist et Ronning, I96I ; O’Deil, et coll., I968). Elles sont liées à une baisse de digestibilité des matières cellulosiques. Celle-ci diminue dans des proportions bien plus faibles quand la luzerne granulée est rationnée comme dans nos expériences, que lorsqu'elle est distribuée ad libitum (DEMARQUILLY et JoURNET, I967). De plus, dans une étude sur vaches laitières, JOURNET et DEMARQUILLY, (I967) observent que la digestibilité de luzerne condensée est seulement légèrement plus basse que celle du foin de luzerne correspondant et que la composition en acides gras volatils a peu varié. Il est probable que ce soit aussi le cas dans nos expériences sur chèvres.

Ce faible effet sur le taux butyreux est à rapprocher de l'engraissement modéré des chèvres qui consomment de la luzerne granulée. Il est en effet rarement plus important que celui des chèvres ingérant du foin de luzerne. Dans nos conditions expérimentales, la distribution de luzerne agglomérée n'a guère permis de dévier de façon apparente le métabolisme lipidique de la chèvre vers l'engraissement, au détriment de la sécrétion des matières grasses du lait comme certains auteurs le suggèrent (MOORE, I965; Col,IN, I968). On peut supposer que la luzerne déshydratée compactée de l'expérience III a quelque peu cette tendance, mais dans des proportions très limitées. 
Par ailleurs, la luzerne déshydratée condensée ou compactée (Expérience III) et le foin de luzerne condensé (Expérience II) tendent à augmenter le taux de matières azotées du lait. JounnE'T et JARRIGE (I969) qui observent le même phénomène sur vaches laitières l'attribuent à une suralimentation énergétique. Dans notre cas, il est difficile d'expliquer de cette manière cette augmentation du taux de ratières azotées, puisque 1'apport énergétique avec les régimes condensés ou compactés n'est pas plus élevé. Journet ( $197^{\circ}$ ) constate aussi ce phénomène sans augmentation de l'énergie ingérée.

Ainsi, dans nos conditions expérimentales, c'est-à-dire pendant des périodes relativement courtes, la luzerne agglomérée semble pouvoir remplacer le foin long ou les tourteaux de l'aliment concentré du régime sans modifier sensiblement les performances laitières des chèvres.

\section{CONSÉQUENCES ZOOTECHNIQUES}

Il apparaît qu'à même niveau d'ingestion de luzerne, qu'elle soit présentée sous forme longue ou agglomérée, les productions laitières sont du même ordre. De ce fait, le niveau de consommation et le risque d'enregistrer des refus, sont des éléments importants à considérer pout orienter le choix d'un type d'alimentation. Par rapport à un foin long de bonne qualité (Expérience III) la consommation de luzerne agglomérée pourra difficilement être supérieure à celle de la luzerne entière, parce que certaines chèvres ont des difficultés à s'habituer à ces fourrages granulés. En revanche, par rapport à un foin de médiocre qualité (Expérience II), le niveau de consommation d'une luzerne agglomérée peut être égal ou même supérieur et l'évolution de la production laitière suit alors celle de consommation alimentaire.

Un broyage grossier paraît plus favorable à une consommation satisfaisante des chèvres qu'un broyage fin. Or, comme le note Demarquil, Y (I968), une luzerne déshydratée qui présente le plus souvent un broyage plus fin que le foin correspondant broyé à la même grille, a une digestibilité plus basse. De tels éléments, indépendamment de considérations économiques, sont plutôt en faveur de l'utilisation de foin aggloméré, que de luzerne déshydratée.

La présence de luzerne granulée dans le régime des chèvres offre certains avantages : elle peut remplacer de façon satisfaisante les tourteaux de l'aliment concentré comme le montre l'expérience I. De plus, elle a tendance à diminuer la consommation d'aliment concentré. Enfin, elle permet une légère augmentation de la teneur en matières azotées du lait qui n'est pas négligeable dans un élevage orienté principalement vers la production fromagère (RICORDEAU et Mocouo'T, I967). Cependant, l'utilisation de luzerne agglomérée doit s'effectuer avec certaines précautions. De la paille de bonne qualité ou un autre fourrage long doit être à la disposition des chèvres pour éviter certains accidents pathologiques (LARVOR, I967; FEHR, résultats non publiés). Par ailleurs, d'après des essais préliminaires sur chèvres (FEHR, résultats non publiés), il ne semble pas, tout comme chez la vache (JOURne'T et HoDEN, I968) que la distribution de tels aliments puissent se réaliser à volonté. L'hétérogénéité des niveaux de consommation entre les individus est alors très importante et les surconsommations sont fréquentes. 
D'après ces résultats, si les conditions économiques le permettent, la constitution de rations pour chèvres laitières à base de paille non broyée, de luzerne agglomérée et de céréales est envisageable. Un tel régime permettrait de faciliter sensiblement la distribution des aliments en élevage caprin. Toutefois, pour s'assurer de son intérêt, il serait souhaitable d'expérimenter ce type de régime sur de longues périodes et notamment pendant une ou plusieurs lactations, afin de connaître les répercussions à long terme de la présence de luzerne granulée dans la ration sur les performances de reproduction, la santé et la production laitière des chèvres.

Rę̧u poù publication en mars 1971.

\section{REMERCIEMENTS}

Nous tenons à remercier M. Legrix, directeur de l'I. T. O. V. I. C., M. de Bordelius, directeur-adjoint de I'I. T. O. V. I. C. et M. Disset, responsable de la Section caprine de l'I. T. O. V. I. C. qui nous ont encouragé dans la réalisation de ce travail, ainsi que M. SAuvant, assistant à l'I. N. R. A. qui a participé à l'interprétation statistique.

\section{SUMMARY}

\section{UTILIZATION OF PEI,LETED FORAGES BY DAIRY GOATS}

During three trials, we studied the effects of pelleted alfalfa hay and pelleted or wafered dehydrated alfalfa as substitutes for oilmeal or normal hay on the behaviour, food intake and milk production and composition in dairy goats. All the diets contained unground straw and were iso-energetic.

The feeding and rumination time decrease by $25 \mathrm{p}$. Ioo in the animals receiving alfalfa pellets, whereas the resting period increase proportionnally. Some goats did not get easily accustomed to pellets. Pelleted or wafered alfalfa tended to increase the intake of straw and to decrease that of concentrates.

The milk yield is little influenced by the physical form of alfalfa and seems to be more affected by the intake level of this forage. There is a tendency for the butterfat percentage to decrease by about $2 \mathrm{~g}$ per $\mathrm{kg}$ milk, and the amount of nitrogenous matter to increase by $\mathrm{I} .5 \mathrm{~g}$ per $\mathrm{kg}$ milk when either pelleted or wafered dehydrated alfalfa is used.

Thus, the alfalfa pellets or wafers + straw can replace the normal hay or oilmeals without very much change in the performance of dairy goats.

\section{RÉFÉRENCES BIBLIOGRAPHIQUES}

Colın P. I968 Citilisation par les ruminants d'aliments agglomérés $S . E . I . \quad$ Étude no 38 réf. P. O. Élev. $37 \mathrm{I}$.

Demarquilly C. 1968. Valeur alimentaire des fourrages déshydratés. Fourrages, nº $36,62-81$.

Demarquilly C., Journet M. I967. Valeur alimentaire des foins condensés. I. Influence de la nature du foin et de la finesse de broyage sur la digestibilité et la quantité ingérée. Ann. Zootech. 16, I23-150.

Fenr P. M., Disset R. 1969. Peut-on alimenter rationnellement les chèvres? Rev. Élevage, $45^{\mathbf{e}}$ $\mathrm{n}^{\circ}$ spécial, I $45^{-15} \mathrm{I}$.

Journet M. I970 Utilisation des aliments condensés par les vaches laitières. Ann. Zootech., 19,85-87. 
Journet M., Demarquilly C. I967. Valeur alimentaire des foins condensés. II. Influence du broyage et de la mise en agglomérés sur la digestion du foin de luzerne dans le rumen. Ann. Zootech., 16, 307-321.

Journet M., Hoden A. Ig68. Utilisation des fourrages déshydratés par les vaches laitières. Fourrages, $\mathrm{n}^{\circ} 36,62-8 \mathrm{I}$.

Journet M., JARrige R. I967. Utilisation des aliments broyés et agglomérés par les bovins. II. Utilisation comparée par la vache laitière du foin de luzerne condensé et du foin de luzerne normal associés à de l'ensilage et des betteraves. Ann. Zootech., 16, 27 I-289.

Keck E., Hale E. W., Schein M. W., Miller R. C. IgG2. A behaviour inventory of sheep fed pelleted and baled alfalfa hay. J.Anim. Sci., 21, I38 (Abstr 6).

LARVOR P. I967. Les fourrages agglomérés et les céréales dans la production de viandc. Accidents entraînés par l'alimentation à base de fourrages agglomérés ou de céréales. Journ. Inf. I. T. C. F. 24-26 janv., $277-288$.

Moore L. A. 1964. Symposium on forage utilization : nutritive value of forage as affected by physical form. I. General principles involved with ruminants and effect of feeding pelleted or wafered forage to dairy cattle. J. Anim. Sci., 23, 230-238.

O'Dell G. D., King W. A., Cook W. C. Ig68. Effect of grinding pelleting and frequency of feeding of forage on fat percentage of milk and milk production of dairy cows. J. Dairy Sci., 51, 50-55.

OWEN F. G. 1969. Value of dehydrated alfalfa in ration for lactating cows. J. Dairy Sci., 52,663-671.

Palmouist I. L., Ronning M. I96I. Some effects caused by fecding ground, pelleted hay as compared to feeding long hay to dairy cows. J. Dairy Sci., 23, 2337 (Abst)r.

Pinot R., Jarrige R. I968. Utilisation des aliments broyés et agglomérés par l'agneau à l'engrais. Ann. Zootech., 17, 5-22.

Ricordeau G., Mocovot G. I967. Influence des variations saisonnières de la composition du lait de chèvre sur le rendement en fromage. Conséquences pratiques pour la sélection. Ann. Zootech., 16, I65-I8I.

Rodrigue C. B., Allen N. N. I960. The effect of fine grinding of hay on ration digestibility, rate of passage and fat content of milk. Can. J. Anim. Sci, 40, 23-29.

Ruckebusch Y., Marguet J. P. I963. Recherches sur le comportement alimentaire chez les ruminants I. Influence de la structure physique des aliments. Rev. Med. Vet., 114, 833-856. 